\title{
Experimental infection of dogs with a Brazilian strain of Rickettsia rickettsii: clinical and laboratory findings
}

\author{
Eliane M Piranda, João Luis H Faccini, Adriano Pinter', Tais B Saito², Richard C Pacheco³, \\ Mitika K Hagiwara ${ }^{2}$, Marcelo B Labruna ${ }^{3 /+}$
}

Departamento de Parasitologia Animal, Instituto de Veterinária, Universidade Federal Rural do Rio de Janeiro, Seropédica, RJ, Brasil ${ }^{1}$ Superintendência de Controle de Endemias, São Paulo, SP, Brasil ${ }^{2}$ Departamento de Clínica Médica ${ }^{3}$ Departamento de Medicina Veterinária Preventiva e Saúde Animal, Faculdade de Medicina Veterinária e Zootecnia, Universidade de São Paulo, Av. Prof. Orlando Marques de Paiva 87, 05508-270 São Paulo, SP, Brasil

The bacterium Rickettsia rickettsii is the etiological agent of an acute, severe disease called Rocky Mountain spotted fever in the United States or Brazilian spotted fever (BSF) in Brazil. In addition to these two countries, the disease has also been reported to affect humans in Mexico, Costa Rica, Panama, Colombia and Argentina. Like humans, dogs are also susceptible to R. rickettsii infection. However, despite the wide distribution of R. rickettsii in the Western Hemisphere, reports of R. rickettsii-induced illness in dogs has been restricted to the United States. The present study evaluated the pathogenicity for dogs of a South American strain of R. rickettsii. Three groups of dogs were evaluated: group 1 (G1) was inoculated ip with R. rickettsii; group 2 (G2) was infested by R. rickettsii-infected ticks; and the control group (G3) was infested by uninfected ticks. During the study, no clinical abnormalities, Rickettsia DNA or R. rickettsii-reactive antibodies were detected in G3. In contrast, all G1 and G2 dogs developed signs of rickettsial infection, i.e., fever, lethargy, anorexia, ocular lesions, thrombocytopenia, anemia and detectable levels of Rickettsia DNA and R. rickettsii-reactive antibodies in their blood. Rickettsemia started 3-8 days after inoculation or tick infestation and lasted for 3-13 days. Our results indicate that a Brazilian strain of R. rickettsii is pathogenic for dogs, suggesting that canine clinical illness due to R. rickettsii has been unreported in Brazil and possibly in the other South American countries where BSF has been reported among humans.

Key word: Rickettsia rickettsii - spotted fever - dog - tick - Brazil

The bacterium Rickettsia rickettsii is the etiological agent of an acute, severe disease called Rocky Mountain spotted fever (RMSF) in the United States or Brazilian spotted fever (BSF) in Brazil. In addition to these two countries, the disease has also been reported to affect humans in Mexico, Costa Rica, Panama, Colombia and Argentina (Paddock et al. 2008). Common clinical signs and symptoms are fever, nausea, vomiting, rash, myalgia, anorexia and headache. The case-fatality rate is $5-10 \%$ in the United States and $10-40 \%$ in Brazil (Galvão et al. 2005, Angerami et al. 2006, Chapman et al. 2006).

Like humans, dogs are also susceptible to $R$. rickettsii infection. However, despite the wide distribution of $R$. rickettsii in the Western Hemisphere, reports of $R$. rickettsii-induced illness in dogs have been restricted to the United States, where the following clinical abnormalities have been observed: fever, lethargy, anorexia, depression, cutaneous petechiae and echymoses, epistaxis, conjunctivitis, ocular discharge, lymph node enlargement, diarrhea, weight loss, dehydration and

Financial support: FAPESP (Grant 06/50918-0 to MBL), CNPq

+Corresponding author: labruna@usp.br

Received 11 June 2008

Accepted 16 September 2008 central nervous system involvement (mostly paraparesis or tetraparesis, ataxia and vestibular syndrome). Hematological abnormalities include anemia, thrombocytopenia and mild leucopenia at the onset of fever followed by leukocytosis (Keenan et al. 1977a, b, Breitschwerdt et al. 1988, Comer 1991). Case-fatality rates in dogs have been reported to be 3\% (Greene 1987), 7\% (Greene et al. 1985) and $0 \%$ in a more recent study (Gasser et al. 2001).

Several serological studies among healthy Brazilian dogs indicate that these animals were infected by $R$. rickettsii, as shown by high antibody titers to $R$. rickettsii, at least four-fold higher than titers to other Rickettsia species known to occur in Brazil (Horta et al. 2004, 2007, Pinter et al. 2008). However, nothing is known about the pathogenicity of South American strains of $R$. rickettsii for dogs. Since ecological differences and genetic polymorphisms between strains of $R$. rickettsii from the United States and South America have been reported (Eremeeva et al. 2003, Karpathy et al. 2007), it is not known how pathogenic South American strains of $R$. rickettsii are for dogs. For this purpose, the present study evaluated experimental infection of dogs with a Brazilian strain of $R$. rickettsii.

\section{MATERIALS AND METHODS}

Eight six-month-old female dogs were used in the study, consisting of five mongrels and three beagles. Dogs were provided by a laboratory animal room, where they were reared with no contact with ticks and under strict sanitary control, being regularly treated with anti- 
worm medication (Pirantel, Febantel, Praziquantel; Vermivet Plus ${ }^{\circledR}$, Biovet, Brazil) and immunized with commercial vaccines for enteroviruses, hepatitis, influenza and leptospirosis (Octa-Cino-Vacin ${ }^{\circledR}$, Biovet, Brazil). During the two weeks before starting the experiment, the dogs were clinically healthy and their paired serum samples (14 days interval) were shown to contain no reactive antibodies to either $R$. rickettsii (serologic reaction performed as described below) or Ehrlichia canis [serologic reaction performed as described elsewhere (Aguiar et al. 2007)].

During the experiment, the dogs were maintained in individual runs in tick-free facilities, where water and commercial food were offered ad libitum. Dogs were assigned to three experimental groups. Group 1 (G1) was composed of three dogs that were each inoculated ip with $\sim 3,000$ Vero cells infected by $R$. rickettsii strain Taiaçu suspended in $1 \mathrm{~mL}$ of sucrose-phosphate-glutamate buffer. The third cell passage was used to inoculate dogs. $R$. rickettsii strain Taiaçu was originally isolated from an Amblyomma aureolatum tick collected on a dog in Taiaçupeba District, Mogi das Cruzes Municipality, state of São Paulo (Pinter \& Labruna 2006a). Group 2 (G2) was composed of three dogs that were each infested by five pairs of $R$. rickettsii-infected $A$. aureolatum adult ticks derived from an experimental colony in our laboratory. Ticks of this colony were experimentally infected by $R$. rickettsii during the previous larval stage by feeding on guinea pigs experimentally infected ip with the third Vero cell passage of strain Taiaçu. The $R$. rickettsii infection of the tick colony was confirmed by testing a random sample of 83 flat nymphs by a PCR protocol targeting a fragment of the rickettsial gene gltA, as previously described (Pinter \& Labruna 2006b). Since all 83 nymphs were PCR positive, the colony was estimated to be $100 \%$ infected. In addition, these nymphs were fed on guinea pigs that developed severe febrile disease exhibiting strong scrotal lesions and high lethality (Pinter \& Labruna 2006b). For tick infestation, white cotton sleeves $(15 \mathrm{~cm} \times 15 \mathrm{~cm})$ were glued to the back of each dog (Kamar heat detector adhesive, Kamar, Steamboat Springs, Co) and the adult ticks were released into the sleeve. The sleeves were opened daily and detached engorged ticks were removed. Group 3 (G3) was composed of two dogs that were left uninfected as a control and each dog in this group was infested by five pairs of uninfected $A$. aureolatum adult ticks derived from a laboratory colony. These uninfected ticks were siblings of the experimentally-infected ticks described above. G1 and G2 each contained one beagle and two mongrel dogs, whereas G3 contained one beagle and one mongrel dog.

Starting from the day of inoculation (G1) or infestation (G2 and G3) of the dogs (designated as day 0 ), each dog was examined daily for rectal temperature and clinical abnormalities until 30 days following inoculation or infestation (DPI). Also, individual blood samples $(0.2 \mathrm{~mL}$ per sample) were collected daily from 0-30 DPI for DNA extraction. Additional blood samples $(2.5 \mathrm{~mL}$ per sample) were collected from each dog every two days from 0-30 DPI, and divided into aliquots; one part solubilized in EDTA for hematology, one part without anticoagulant to separate serum for serology, and a third part used to inoculate guinea pigs ip $(0.5 \mathrm{~mL}$ per guinea pig), with two guinea pigs being inoculated per dog at each time point. Rectal temperature was measured daily in each guinea pig until 21 DPI, but if a guinea pig presented at least two consecutive days of high fever (temperature $>40^{\circ} \mathrm{C}$ ) within this period, it was euthanized and its spleen was collected and processed for DNA extraction and PCR for rickettsial DNA, as described below for canine blood. Every seven days from 31-90 DPI and every 14 days from 91-180 DPI, canine blood samples were collected without anticoagulant to separate serum for serology testing.

For hematology, blood cells were counted in an automatic counter (Horiba ABX Brasil, São Paulo, Brazil) to determine total number of red blood cells, white blood cells, and platelets, hemoglobin level and globular volume.

DNA from canine blood $(200 \mu \mathrm{L}$ of whole blood from each sample) was extracted using the DNeasy Tissue Kit (Qiagen, Chatsworth, CA, USA) following the manufacturer's protocols. All blood samples were individually processed by a real-time PCR assay with primers CS-5 (5' AGAGAAAATTATATCCAAATGTTGAT-3') and CS-6 (5' -AGGGTCTTCGTGCATTTCTT-3'), designed to amplify a $147 \mathrm{bp}$ fragment of the citrate synthase gene ( $\mathrm{gltA}$ ) of Rickettsia spp. A fluorogenic probe [5' 6-FAM d (CATTGTGCCATCCAGCCTACGGT) BHQ-1 3'] (Integrated DNA Technologies, San Diego, CA, USA) positioned $76 \mathrm{bp}$ downstream of the forward primer and $3 \mathrm{bp}$ upstream of the reverse primer was used in the reactions (Labruna et al. 2004). Real-time PCRs were performed in a 7500 Real Time PCR Systems apparatus (Applied BioSystems, Foster City, CA, USA) with $25 \mu \mathrm{L}$ per reaction, which contained $2.5 \mu \mathrm{L}$ of an individual blood DNA template, $2.5 \mu \mathrm{L}$ of PCR buffer (10 X), $4 \mu \mathrm{L}$ of deoxynucleotide triphosphates mixture $(1.25 \mathrm{mM}), 1.0 \mu \mathrm{L}$ $\mathrm{MgCl}_{2}(50 \mathrm{mM}), 0.1 \mu \mathrm{L}$ of Platinum Taq DNA polymerase $(5,000 \mathrm{U} / \mathrm{mL})$ (Invitrogen, São Paulo, Brazil), $1 \mu \mathrm{L}$ of each primer at $10 \mu \mathrm{M}, 0.25 \mu \mathrm{L}$ of the probe at $20 \mu \mathrm{M}$ and bi-distilled water to a final volume of $25 \mu \mathrm{L}$. Realtime PCR cycling conditions were as described by Labruna et al. (2004). This combination of primers and probe has shown a sensitivity down to 1 DNA copy of $R$. rickettsii (Labruna et al. 2004). For each reaction, five negative controls $(2.5 \mu \mathrm{L}$ of the same bi-distilled water mentioned above) and a positive control (300 ng of DNA of Rickettsia parkeri-infected Vero cells) were included.

Blood serum samples collected from days 0-180 DPI were tested by an indirect immunofluorescence assay (IFA) using a $R$. rickettsii strain Taiaçu crude antigen and a fluorescein isothiocyanate-labeled rabbit anti-dog IgG (Sigma, St Louis, USA), as previously described (Labruna et al. 2007). Canine sera were serially diluted in two-fold increments with PBS starting from 1:64 dilution. For each serum sample, the endpoint titer reacting with $R$. rickettsii was determined. In each slide, a serum previously shown to be non-reactive (negative control) and a known reactive serum (positive control) were tested at the 1:64 dilution. The geometric mean for each titer of the three groups was obtained as previously de- 
scribed (Breitschwerdt et al. 1988). Additionally, canine sera from 24 and 180 DPI were tested by IFA against six Rickettsia species (R. rickettsii, R. parkeri, Rickettsia amblyommii, Rickettsia rhipicephali, Rickettsia felis, Rickettsia bellii) known to occur in Brazil (Labruna et al. 2007), in order to compare endpoint titers against homologous $(R$. rickettsii) and heterologous antigens (remaining Rickettsia species).

The present study was previously approved by the Bioethical Committee in Animal Research of the Faculty of Veterinary Medicine of the University of São Paulo (protocol 913/2006).

\section{RESULTS}

During the study, dogs from the control group (G3) showed neither clinical nor hematological abnormalities associated with rickettsial infection, nor were there any detectable Rickettsia DNA or reactive antibodies in their blood. In contrast, all dogs from both the G1 (inoculated via ip with $R$. rickettsii) and G2 (infected through infestation with $R$. rickettsii-infected ticks) developed signs of rickettsial infection, i.e., they showed clinical and hematological abnormalities and detectable levels of Rickettsia DNA (rickettsemia) and reactive antibodies in their blood (Figs 1-3). Additionally, guinea pigs inoculated with G1 and $\mathrm{G} 2$ canine blood from 2-14 DPI developed fever within 48-96 $\mathrm{h}$ and, after being euthanized, their spleens were shown to contain rickettsial DNA. No guinea pig inoculated with blood from G3 dogs developed fever.

Clinical indications among dogs from both groups G1 and G2 were similar, except for one G2 dog, which presented ocular lesions characterized by bilateral ocular discharge, scleral congestion and conjunctival edema. Dogs from the G1 presented rickettsemia, anorexia, fever (rectal temperature $>39.5^{\circ} \mathrm{C}$ ) and lethargy earlier than $\mathrm{G} 2$ dogs (Fig. 1). In each group, clinical signs generally occurred within the febrile period. Thus, rickettsemia, defined as the presence of rickettsial DNA detected in dog blood

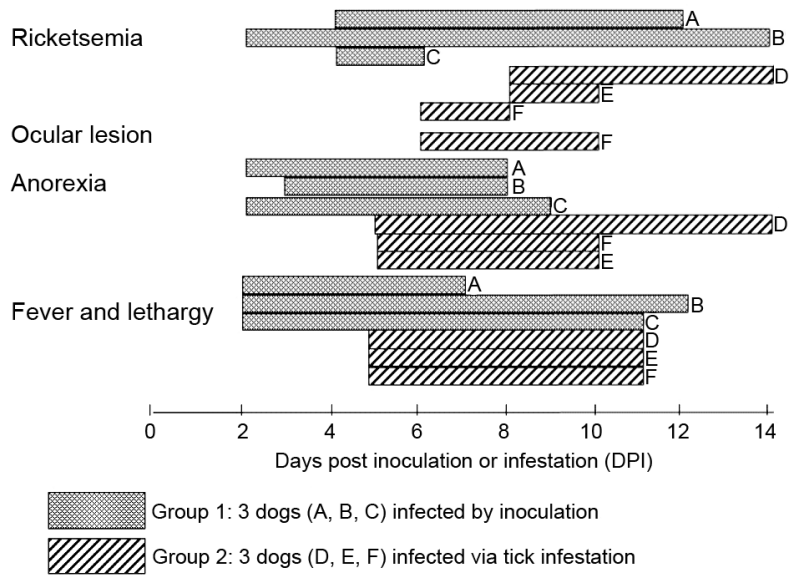

Fig. 1: selected clinical findings and rickettsemia duration for dogs of two experimental groups (G1 and G2) exposed to Rickettsia rickettsii infection. G1 consisted of three $\operatorname{dogs}(\mathrm{A}, \mathrm{B}$ and $\mathrm{C})$ inoculated ip with $R$. rickettsii; $\mathrm{G} 2$ consisted of three dogs $(\mathrm{D}, \mathrm{E}$ and $\mathrm{F})$ infested with $R$. rickettsii-infected ticks. by our real-time PCR assay, was first detected at 2 DPI in G1 dogs and at 6 DPI in G2 dogs. The duration of rickettsemia in infected dogs was limited, ranging from three days (dogs C, E and F) to 13 days (dog B) (Fig. 1).

Regarding hematologic changes, hemoglobin concentration, package cell volume and platelet counts decreased below the reference range for dogs during the febrile period in both G1 and G2, with values remaining lower for up to six days after the febrile period in G2 dogs (Fig. 2). No marked change in erythrocyte or leukocyte counts was observed. Clinical and hematologic abnormalities resolved in G1 and G2 dogs without sequelae. No dog presented hemorrhagic (including cutaneous or mucous), locomotor or neurological signs during the study, during which no antibiotic was administrated.

During the study, G3 dogs remained seronegative to $R$. rickettsii at the 1:64 serum dilution. The antibody response to $R$. rickettsii was first documented on DPI 4 for G1 dogs and DPI 8 for G2 dogs (Fig. 3). Mean peak titers were reached by DPI 24 or 44 for G1 or G2 dogs, respectively. Mean serum titers to $R$. rickettsii essentially remained stable through 114 DPI for G2 and then decreased slowly through 180 DPI. Despite the higher and earlier peak titers observed in G1 dogs, their mean titer decreased slowly in a pattern similar to that of G2 dogs.

For G1 dogs, individual sera reached peak titers of $32,768-65,536$ to $R$. rickettsii, and then decreased slowly 3-5 dilutions through 180 DPI, when titers ranged from $2,048-4,096$. For G2 dogs, individual sera reached peak titers of 32,768 for all three dogs, and then decreased slowly 2-4 dilutions through 180 DPI, when titers ranged from 2,048-8,192.

Canine sera from 24 and 180 DPI tested against six rickettsial antigens all showed higher titers to $R$. rickettsii than to R. parkeri, R. amblyommii, $R$. rhipicephali, $R$. felis and $R$. bellii, or the same titer for both R. rickettsii and $R$. parkeri in one dog at 180 DPI (Table). In four dogs at 24 DPI and two dogs at 180 DPI titers to $R$. rickettsii were at least four-fold higher to $R$. rickettsii than to the remaining Rickettsia species.

\section{DISCUSSION}

In the present study, dogs became ill after exposure to strain Taiaçu of $R$. rickettsii through both ip inoculation of the agent or via the parasitism of $R$. rickettsiiinfected ticks. This latter exposure method mimicked natural conditions in some areas of the state of São Paulo, where dogs are exposed to $R$. rickettsii-infected A. aureolatum ticks, a known vector of the disease in humans (Pinter et al. 2004, Pinter \& Labruna 2006a). Thus, our results indicate that a Brazilian strain of $R$. rickettsii is pathogenic for dogs, despite the fact there has been no report of clinical illness caused by $R$. rickettsii among Brazilian dogs, including all areas where BSF has been reported in humans.

Experimental studies on canine RMSF have shown that the severity of the induced illness is related to the infectious dose of $R$. rickettsii (Keenan et al. 1977a, b). In those studies, dogs were inoculated intravenously with gradually increasing doses of yolk sac suspensions of $R$. rickettsii, which resulted in fatalities among dogs receiv- 

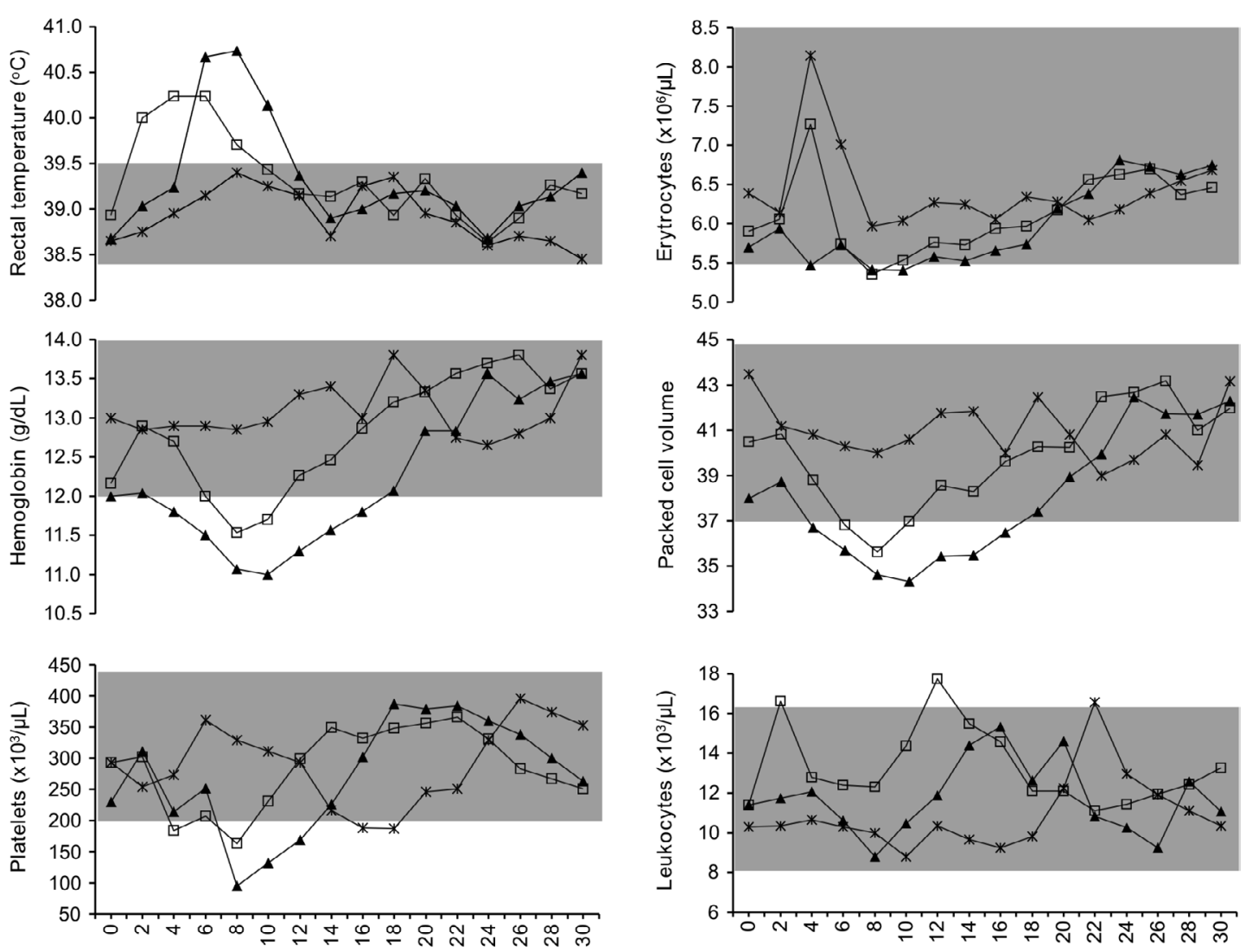

Days post inoculation or infestation

Fig. 2: mean values of rectal temperature and hematologic values for dogs of three experimental groups (G1, G2 and G3) evaluated during 30 days after exposure to Rickettsia rickettsii: G1 ( $\square \square)$ consisted of three dogs inoculated ip with R. rickettsii; G2 ( $-\mathbf{\Lambda})$ consisted of three dogs infested with $R$. rickettsii-infected ticks; G3 (x-x) consisted of two dogs infested with uninfected ticks, being the uninfected control group. Shaded areas represent laboratory reference range in the Veterinary Hospital of the University of São Paulo.

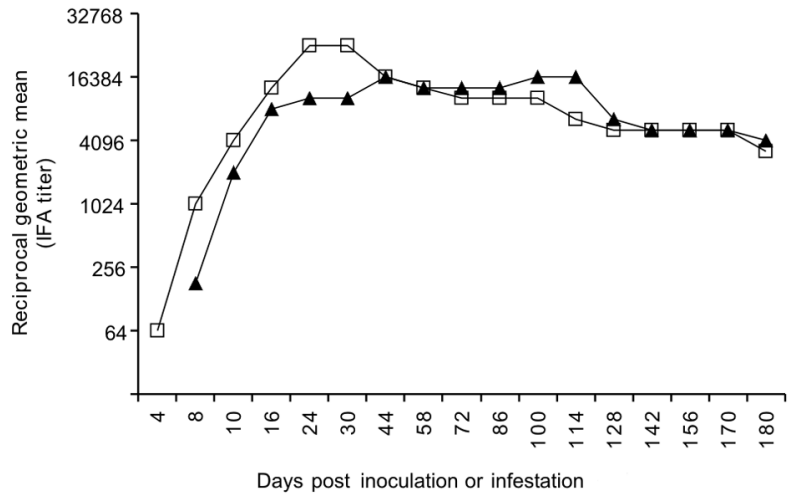

Fig. 3: serologic response of dogs of two experimental groups (G1 and G2) to Rickettsia rickettsii antigen by the indirect immunofluorescence assay (IFA). G1 ( $\square-\square)$ consisted of three dogs inoculated ip with $R$. rickettsii; G2 ( $-\mathbf{\Delta})$ consisted of three dogs infested with $R$. rickettsii-infected ticks.

ing higher doses (Keenan et al. 1977a, b). In the present study, G1 dogs were inoculated ip with a very high dose of tissue-cultured $R$. rickettsii $\approx \approx 3,000$ infected Vero cells), whereas G2 dogs were infected via tick parasit- ism (at most 10 infected adult ticks per dog). Since the onset of fever and rickettsemia occurred at least three days later in G2 dogs in comparison to G1 dogs (Fig. 1), one might suppose that these latter dogs were exposed to a higher $R$. rickettsii-infective dose, which should have induced a more severe disease. However, our results showed that G2 dogs (infected via ticks) developed a slightly more severe illness, with higher rectal temperatures and lower platelet, packed cell volume and hemoglobin values, all of which lasted days longer than in G1 dogs (Fig. 2). In addition, the only dog presenting ocular lesions belonged to G2. The causes for such differences could be related to mode of acquisition of the $R$. rickettsii infection. While G1 dogs received a single, but high infectious dose at DPI 0, G2 dogs might have received lower infectious doses over several consecutive days during tick engorgement. In addition, disease severity in G2 dogs could be affected by the tick-passage of strain Taiaçu since G1 dogs were inoculated directly with the third in vitro passage.

Dogs of the present study presented rickettsemia (as determined by detectable rickettsial DNA by real-time PCR and by inoculation of guinea-pigs with canine blood) lasting from 3-13 days (mean $=8.3$ days for G1, 
TABLE

Endpoint antibody titers by indirect immunofluorescence assay (IFA) to six Rickettsia species in dogs from Group 1 (G1) (A, B and C) and Group 2 (G2) (D, E and F) at 24 and 180 days post inoculation or infestation (DAI). G1 dogs were inoculated ip with $R$. rickettsii and $\mathrm{G} 2$ dogs were infested with $R$. rickettsii-infected ticks

\begin{tabular}{|c|c|c|c|c|c|c|}
\hline \multirow[b]{2}{*}{ Dog (DAI) } & \multicolumn{6}{|c|}{ IFA endpoint titer to the following rickettsial antigens } \\
\hline & R. rickettsii & R. parkeri & R. amblyommii & R. rhipicephali & R. felis & R. bellii \\
\hline A (24) & 32768 & 4096 & 4096 & 1024 & 512 & 512 \\
\hline B (24) & 16384 & 8192 & 4096 & 1024 & 1024 & 2048 \\
\hline $\mathrm{C}(24)$ & 65536 & 32768 & 4096 & 1024 & 1024 & 1024 \\
\hline $\mathrm{D}(24)$ & 16384 & 4096 & 2048 & 1024 & 256 & 512 \\
\hline E (24) & 8192 & 2048 & 2048 & 2048 & 256 & 512 \\
\hline F (24) & 8192 & 2048 & 1024 & 512 & 256 & 512 \\
\hline A (180) & 4096 & 2048 & 512 & 512 & $<64$ & $<64$ \\
\hline B (180) & 4096 & 4096 & 2048 & 2048 & 128 & 128 \\
\hline C (180) & 2048 & 1024 & 1024 & 512 & 256 & 512 \\
\hline D (180) & 2048 & 512 & 512 & 128 & 128 & 128 \\
\hline E (180) & 4096 & 1024 & 512 & 512 & $<64$ & 64 \\
\hline F (180) & 8192 & 4096 & 256 & 64 & 64 & 64 \\
\hline
\end{tabular}

4.3 days for $\mathrm{G} 2$ ). These periods are similar to those reported by Norment and Burgdorfer (1984), who found rickettsemia (determined by meadow vole inoculation) lasting from 3-7 days, with mean rickettsemia periods lasting for 4.3 or 6.3 days when dogs were infected by $R$. rickettsii via ip injection with yolk sac suspension or via parasitism of infected Dermacentor andersoni ticks, respectively. Since the real-time PCR assay employed in the present study has been shown to detect as few as one copy of the $R$. rickettsii genome (Labruna et al. 2004), this technique seems to be useful for the determination of rickettsemia in animal blood.

Both G1 and G2 dogs developed high $R$. rickettsiiantibody titers, which remained high $(\geq 2,048)$ at 180 DPI. Although Keenan et al. (1977b) detected R. rickettsii-reactive antibodies in dogs 12 months after experimentally induced infection, it is not known how long antibodies persist in dogs (Magnarelli et al. 1982). Therefore, seropositivity may not necessarily indicate a very recent infection. On the other hand, long periods of seroreactivity highlight the important role of dogs as sentinels for surveillance of RMSF or BSF (Sexton et al. 1976, Lemos et al. 1996, Paddock et al. 2002, Sangioni et al. 2005) since a seroreactive dog indicates circulation of spotted fever group rickettsia in a given area, at least within the previous 6-12 months.

Serological tests at 24 DPI (around peak titers) and 180 DPI (end of study) showed higher endpoint titers to $R$. rickettsii (homologous reaction) than to the other five Rickettsia species (heterologous reaction) known to occur in Brazil, except for one dog at 180 DPI that showed the same titer for both $R$. rickettsii and $R$. parkeri. Previous canine serology surveys in Brazil used the criteria that when a serum displayed an endpoint titer for a given Rickettsia species at least four-fold higher than that observed for any other Ricketttsia species, that serum would be considered homologous to the first Rickettsia species or to a very closely related genotype (Horta et al. 2004, 2007,
Labruna et al. 2007, Pinter et al. 2008). This assumption is supported by the present study since no dog showed a higher titer to heterologous antigens, and six sera showed titers to $R$. rickettsii at least four-fold higher than the titers to the remaining Rickettsia species (Table).

The results of the present study strongly suggest that canine clinical illness due to $R$. rickettsii has gone unreported in Brazil. It is possible that some of these cases have been misidentified as canine monocytic ehrlichiosis (CME), a highly prevalent tick-borne disease in Brazil that is caused by E. canis (Aguiar et al. 2007), which induces clinical and hematologic symptoms in dogs very similar to those caused by $R$. rickettsii (Greene et al. 1985, Grindem et al. 1999).

Finally, Brazilian veterinary clinicians should include $R$. rickettsii infection in a differential diagnosis of CME and other acute nonspecific febrile illness of dogs, especially because $R$. rickettsii is highly pathogenic for humans. It is likely that the occurrence of illness due to $R$. rickettsii in a pet dog means that there is a risk of contracting the disease by humans living in the same residence (in close contact with ticks). For instance, there have been several reports of concurrent RMSF in dogs and their owners (Paddock et al. 2002, Elchos \& Goddard 2003). Therefore, proper diagnosis of BSF in dogs might alert owners to take measures to prevent the occurrence or even the lethality of the disease in humans. Due to the persistence of $R$. rickettsii-reactive antibodies in infected dogs for at least 6-12 months, dog-directed serologic surveys may alert owners that the pathogen is endemic to their immediate environment and also provide doctors with a presumptive diagnosis for dog owners presenting with unexplained febrile illness (Paddock et al. 2002).

\section{ACKNOWLEDGMENTS}

To Laboratório Biovet, Brazil, for providing tick-naïve dogs for the present study, and the anonymous reviewers and editors, for their valuable suggestions to improve this manuscript. 


\section{REFERENCES}

Aguiar DM, Cavalcante GT, Pinter A, Gennari SM, Camargo LMA, Labruna MB 2007. Prevalence of Ehrlichia canis (Rickettsiales: Anaplasmataceae) in dogs and Rhipicephalus sanguineus (Acari: Ixodidae) ticks from Brazil. J Med Entomol 44: 126-132.

Angerami RN, Resende MR, Feltrin AF, Katz G, Nascimento EM, Stucchi RS, Silva LJ 2006. Brazilian spotted fever: a case series from an endemic area in Southeastern Brazil: clinical aspects. Ann N Y Acad Sci 1078: 252-254.

Breitschwerdt EB, Walker DH, Levy MG, Burgdofer W, Cobertt WT, Hurlbert SA, Stebbins ME, Curtins BC, Allen DA 1988. Clini$\mathrm{cal}$, hematologic and humoral immune response in female dogs inoculated with Rickettsia rickettsii and Rickettsia montana. Am $J$ Vet Res 49: 70-76.

Chapman AS, Murphy SM, Demma LJ, Holman RC, Curns AT, McQuiston JH, Krebs JW, Swerdlow DL 2006. Rocky Mountain spotted fever in the United States, 1997-2002. Ann N Y Acad Sci 1078: 154-155.

Comer KM 1991. Rocky Mountain spotted fever. Vet Clin North Am Small Anim Pract 21: 27-48.

Elchos BN, Goddard J 2003. Implications of presumptive fatal Rocky Mountain spotted fever in two dogs and their owner. $J$ Am Vet Med Assoc 223: 1450-1452.

Eremeeva ME, Klemt RM, Santucci-Domotor LA, Silverman DJ, Dasch GA 2003. Genetic analysis of isolates of Rickettsia rickettsii that differ in virulence. Ann NY Acad Sci 990: 717-722.

Galvão MA, Silva LJ, Nascimento EM, Calic SB, Sousa R, Bacellar F 2005. Rickettsial diseases in Brazil and Portugal: occurrence, distribution and diagnosis. Rev Saude Publica 39: 850-856.

Gasser AM, Birkenheuer AJ, Breitschwerdt EB 2001. Canine Rocky Mountain spotted fever: a retrospective study of 30 cases. $J \mathrm{Am}$ Anim Hosp Assoc 37: 41-48.

Greene CE 1987. Rocky Mountain spotted fever. J Am Vet Med Assoc 191: 666-671.

Greene CE, Burgdorfer W, Cavagnolo R, Philip RN, Peacock MG 1985. Rocky Mountain spotted fever in dogs and its differentiation from canine ehrlichiosis. J Am Vet Med Assoc 186: 465-472.

Grindem CB, Breitschwerdt EB, Perkins PC, Cullins LD, Thomas TJ, Hegarty BC 1999. Platelet-associated immunoglobulin (antiplatelet antibody) in canine Rocky Mountain spotted fever and Ehrlichiosis. J Am Anim Hosp Assoc 35: 56-61.

Horta MC, Labruna MB, Pinter A, Linardi PM, Schumaker TTS 2007. Rickettsia infection in five areas of the state of São Paulo. Mem Inst Oswaldo Cruz 102: 793-801.

Horta MC, Labruna MB, Sangioni LA, Vianna MCB, Gennari SM, Galvão MA, Mafra CL, Vidotto O, Schumaker TT, Walker DH 2004. Prevalence of antibodies to spotted fever group rickettsiae in humans and domestic animals in a Brazilian spotted fever endemic area in the state of São Paulo, Brazil: serological evidence for infection by Rickettsia rickettsii and another spotted fever group Rickettsia. Am J Trop Med Hyg 71: 93-97.

Karpathy SE, Dasch GA, Eremeeva ME 2007. Molecular typing of isolates of Rickettsia rickettsii by use of DNA sequencing of variable intergenic regions. J Clin Microbiol 45: 2545-2553.
Keenan KP, Buhles WC, Huxsoll Jr DL, Williams RG, Hildebrandt PK 1977a. Studies on the pathogenesis of Rickettsia rickettsii in the dog: clinical and clinicopathologic changes of experimental infection. Am J Vet Res 38: 851-856.

Keenan KP, Buhles WC, Huxsoll Jr DL, Williams RG, Hildebrandt PK, Campbell JM, Stephenson EH 1977b. Pathogenesis of infection with Rickettsia rickettsii in the dog: a disease model for Rocky Mountain spotted fever. J Infect Dis 135: 911-917.

Labruna MB, Horta MC, Aguiar DM, Cavalcante GT, Pinter A, Gennari SM, Camargo LMA 2007. Prevalence of Rickettsia infection in dogs from the urban and rural areas of Monte Negro municipality, Western Amazon, Brazil. Vector Borne Zoonotic Dis 7: 249-255.

Labruna MB, Whitworth T, Horta MC, Bouyer DH, McBride JW, Pinter A, Popov V, Gennari SM, Walker DH 2004. Rickettsia species infecting Amblyomma cooperi ticks from an area in the state of São Paulo, Brazil, where Brazilian spotted fever is endemic. $J$ Clin Microbiol 42: 90-98.

Lemos ERS, Machado RD, Coura JR, Guimarães MAA, Chagas N 1996. Epidemiological aspects of the Brazilian spotted fever: serological survey of dogs and horses in an endemic area in the state of São Paulo, Brazil. Rev Inst Med Trop Sao Paulo 38: 427-430.

Magnarelli LA, Anderson JF, Philip RN 1982. Antibodies to spotted fever group rickettsiae in dogs and prevalence of infected ticks of Southern Connecticut. Am J Vet Res 43: 656-659.

Norment BR, Burgdorfer W 1984. Susceptibility and reservoir potential of the dog to spotted fever-group rickettsiae. Am J Vet Res 45: 1706-1710.

Paddock CD, Brenner O, Vaid C, Boyd DB, Berg JM, Joseph RJ, Zaki SR, Childs JE 2002. Short report: concurrent Rocky Mountain spotted fever in a dog and its owner. Am J Trop Med Hyg 66: 197-199.

Paddock CD, Fernandez S, Echenique GA, Sumner JW, Reeves WK, Zaki SR, Remondegui CE 2008. Rocky Mountain spotted fever in Argentina. Am J Trop Med Hyg 78: 687-692.

Pinter A, Dias RA, Gennari SM, Labruna MB 2004. Study of the seasonal dynamics, life cycle and host specificity of Amblyomma aureolatum (Acari: Ixodidae). J Med Entomol 41: 324-332.

Pinter A, Horta MC, Pacheco RC, Moraes-Filho J, Labruna MB 2008. Serosurvey for Rickettsia spp in dogs and humans from a Brazilian spotted fever endemic area in the state of São Paulo. Cad Saude Publ 24: 247-252.

Pinter A, Labruna MB 2006a. Isolation of Rickettsia rickettsii and Rickettsia bellii in cell culture from the tick Amblyomma aureolatum in Brazil. Ann NY Acad Sci 1078: 523-529.

Pinter A, Labruna MB 2006b. Competência vetorial de Amblyomma aureolatum na transmissão da bacteria Rickettsia rickettsii. In Proceedings of the 2nd Simpósio Latino-Americano de Rickettsioses, Ribeirão Preto, Brazil, p. 368.

Sangioni LA, Horta MC, Vianna MCB, Gennari SM, Soares RS, Galvão MAM, Schumaker TTS, Ferreira F, Vidotto O, Labruna MB 2005. Rickettsial infection in animals and Brazilian spotted fever endemicity. Emerg Infect Dis 11: 265-270.

Sexton DJ, Burgdorfer W, Thomas L, Norment BR 1976. Rocky Mountain spotted fever in Mississippi: survey for spotted fever antibodies in dogs and for spotted fever group rickettsiae in dog ticks. Am J Epidemiol 103: 192-197. 